\title{
El punto de vista de los pacientes sobre la seguridad clínica de los hospitales. Validación del Cuestionario de Percepción de Seguridad
}

\author{
José Joaquín Mira ${ }^{1,2}$, Susana Lorenzo ${ }^{3}$, Julián Vitaller ${ }^{4,5}$, \\ Medhi Ziadi ${ }^{6}$, Lidia 0 rtiz $^{2}$, Emilio Ignacio ${ }^{7}$, Jesús Aranaz ${ }^{4,5}$. \\ Hospital clinical safety from the \\ patient's point of view. Validation of \\ a safety perception questionnaire
}

Background: Approximately 10\% of hospitalized patients suffer an adverse event during their hospital stay. An important proportion of subjects also feel that they have a high risk of suffering such an event during an eventual hospitalization. Aim: To determine the perception on clinical safety among patients discharged from a hospital. Material and methods: A questionnaire about hospital safety was mailed to 1300 patients discharged from a hospital. The questionnaire was analyzed using construct validity, predictive validity and Cronbach Alpha for internal consistency. Results: The questionnaire was answered by 384 patients, yielding a response rate of $29 \%$. Of these, 77 incomplete answers were discarded. Thirty one subjects (10\%) reported a possible adverse event. In 19 cases (5.8\%), it was due to medication errors and in $19(6.1 \%)$, to surgical procedures. In seven cases $(2.3 \%)$ both errors coincided (2.3\%). According to the predictive validity of the questionnaire, if a patient reports an adverse event, the confidence in the hospital and in the professionals is reduced $(p<0.001)$, communication with the physician is considered inappropriate $(p=0.0001)$ and risk perception increases $(p=0.003)$. Unsatisfied patients are those that believe that they have higher risks of suffering a medical error $(p=0.005)$. Conclusions: Risk perception for adverse events increases after having suffered such an event. Patient satisfaction minimizes the effects of adverse events on their confidence and attitude (Rev Méd Chile 2009; 137: 1441-8).

(Key words: Management, medical practice; Medication therapy management; Personal satisfaction; Safety)

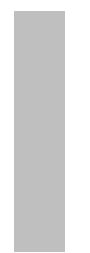

Recibido el 6 de mayo, 2009. Aceptado el 4 de octubre, 2009.

Estudio financiado por el FIS, referencias PI06-90043 e IP-60574.

${ }^{1}$ Centro de Salud Hospital-Plá, Departamento de Salud 17. Alicante. ${ }^{2}$ Departamento de Psicología de la Salud, Universidad Miguel Hernández. Elche. ${ }^{3}$ Fundación Hospital Alcorcón, Madrid. ${ }^{4}$ Departamento de Salud Pública, Historia Medicina y Obstetricia, Universidad Miguel Hernández. Elche. ${ }^{5}$ Hospital General Universitario de Alicante. ${ }^{6} \mathrm{Hospital}$ General Universitario de Elche. ${ }^{7}$ Universidad de Cádiz, España.

Correspondencia a: Dr. José Joaquín Mira. Universidad Miguel Hernández de Elche. Edificio Rectorado, Avd. Universidad s/n, Elche 03202. Teléfono: 966658984. E mail: jose.mira@umh.es 
$\mathrm{L}^{\mathrm{a}}$ a mayoría de ciudadanos minimizan la probabilidad de sufrir un efecto adverso (EA). Sin embargo, esa probabilidad no es insignificante ${ }^{1}$. En España, 8,4\% (IC 95\%: 7,7\%-9,1\%) de los pacientes hospitalarios ${ }^{2}$ y 10,1\%o (IC 95\%: 9,5\%o$10,7 \%$ ) en atención primaria ${ }^{3}$, sufren un EA.

El estudio de los EA desde la perspectiva de los pacientes se justifica porque minan la confianza en los profesionales ${ }^{4}$ y dificultan la relación médico-paciente ${ }^{5,6}$.

Los estudios sobre percepción de seguridad clínica o bien identifican la frecuencia de EA; 0 bien indagan si se sienten "seguros" los pacientes. Entre 6\% y 51\% 4,7-12 informan de un EA, dependiendo del tipo de pregunta (y si incluye a familiares) y del espacio de tiempo (desde un año hasta "toda la vida").

El $9 \%$ de europeos ${ }^{11}, 5 \%$ australianos ${ }^{13}$ y $3 \%$ canadienses ${ }^{12}$ creen que es alta la probabilidad de sufrir un error. En Estados Unidos de Norteamérica (al menos en 2000) la preocupación por la seguridad clínica era mayor que la inquietud por la seguridad aérea. Para $31 \%$ de españoles ${ }^{15}$ se producen "bastantes" errores clínicos y $12 \%{ }^{6}$ consideran que el riesgo de un error quirúrgico es alto.

El objetivo de este estudio es establecer una medida con la que analizar la percepción de seguridad que tienen los pacientes que hablan español tras el alta hospitalaria.

\section{Material y MÉTOdO}

Estudio observacional, de validación de un cuestionario de percepción de seguridad de pacientes hospitalarios. El estudio de campo se realizó en 2008. Primero, se identificaron las dimensiones del concepto "seguridad" y, posteriormente, sus propiedades métricas.

Para definir los contenidos se revisó la literatu$\mathrm{ra}^{16}$ (referentes: Eurobarómetro ${ }^{11}$, Fundación Kai$\operatorname{ser}^{14}$ y encuesta de seguridad de Alberta ${ }^{12}$ ) y se aplicó técnica grupo Nominal con representantes de asociaciones de pacientes ${ }^{17}$. Se incluyeron cuatro dimensiones (validez de contenido): confianza en el médico y enfermero; comunicación positiva con los profesionales; percepción de seguridad ante determinados riesgos de la vida cotidiana; y capacidad personal para intervenir y prevenir riesgos.
Un panel de 5 profesionales redactó preguntas tipo, poniendo a prueba mediante sucesivas pruebas su comprensión. El cuestionario inicial contó con 30 preguntas. Adicionalmente, se preguntaba por el nivel de satisfacción y si había padecido EA en la última hospitalización. En la redacción de estas preguntas se tuvieron en cuenta resultados previos $^{17}$ que ponían de manifiesto la dificultad de los pacientes para hablar de EA y su confusión respecto de lo que constituye un error clínico.

Sujetos. Se invitó a contestar a 1.300 pacientes a los 15-20 días del alta. No se realizó ningún recordatorio para garantizar anonimato del informante. El estudio fue aprobado por el Comité Ético de Investigación.

El tamaño muestral fue calculado para un error máximo de $5 \%, p=q=0,50$, y $\alpha$ de $95 \%$. Se recurrió a un muestreo aleatorio con afijación proporcional, considerando el número de altas del año anterior y realizando dos cortes a lo largo del año. El tamaño muestral se incrementó para una esperanza de respuesta de $30 \%$.

Estadística. Se analizó la no respuesta y fallos de comprensión. Se descartaron 4 preguntas (riesgo de accidentes aéreos o ferroviarios) debidos a su escasa probabilidad para los encuestados. Se han analizado validez (de construcción y predictiva) y consistencia interna ${ }^{18}$. La validez de constructo se ha estimado mediante análisis factorial de Componentes Principales y rotación Varimax. Adicionalmente, se sometió a este mismo procedimiento las puntuaciones directas en cada uno de los factores (análisis factorial de segundo orden). Se calculó KMO y el test de esfericidad de Bartlett. En estos análisis se ha asumido que las respuestas pueden tratarse como escala de intervalo.

Se ha recurrido a Alpha de Cronbach para estimar la consistencia interna. Adicionalmente, se han considerado el valor del Alpha al eliminar la pregunta de la escala y su valor propio en el análisis de Componentes Principales. Para determinar la validez predictiva se ha recurrido a $\mathrm{Chi}$ Cuadrado, aplicando la corrección de Yates en el caso de tablas $2 \times 2$, considerando edad, sexo y la satisfacción del paciente como variables de ajuste. En sucesivos análisis se ha considerado como variable dependiente la frecuencia con la que se informa de un EA. De forma convencional se ha 
considerado que existen diferencias significativas cuando $\mathrm{p}<0,05$, para un $\alpha$ de $95 \%$.

Para determinar la utilidad del cuestionario se contrastaron las siguientes hipótesis. Primero, la ocurrencia de EA hace disminuir la confianza del paciente en profesionales y sistema sanitario. Segundo, cuando se informa un EA se deteriora la comunicación con el médico. Tercero, cuando se sufre un EA se experimenta reacción de alerta en futuras hospitalizaciones.

\section{Resultados}

Respondieron 384 pacientes (tasa de respuesta de $29,5 \%$ ). Setenta y siete casos fueron descartados para los posteriores análisis al contestar de forma incompleta a la encuesta, por lo que el error de precisión fue 5,6\%. El 49,3\% eran mujeres, 5,4\% tenía menos de 30 años y 53,8\% más de 60 años.

En la muestra consultada 10,1\% (IC 95\% 9,8$19,3)$ informan que "algo no fue bien del todo", por lo que precisaron un tratamiento adicional (posible EA en 31 de 307 casos). En 19 casos (5,8\%, IC 95\% $4,5-6,1)$ los encuestados lo atribuyeron a errores a la medicación y en otros 19 (6,1\%, IC 95\% 5,8-6,4) a la intervención quirúrgica. El 21,1\% de los supuestos errores de medicación y $26,3 \%$ de los quirúrgicos tuvieron, según los encuestados, graves consecuencias (Figura 1). En 7 casos el paciente informó sufrir ambos errores (2,3\%, IC 95\% 2,0-2,6). La frecuencia de EA fue similar entre hombres y mujeres y entre los distintos grupos de edad.

Se identifican 8 factores (Tabla 1) que explican $64,1 \%$ de la varianza. Únicamente en un caso (errores debidos a insuficientes medios) el valor propio del elemento es inferior a 0,5. Tres elementos saturan de forma significativa en más de un factor, aunque, salvo en el caso de la pregunta sobre la capacidad para evitar un accidente de tráfico, en el resto claramente saturan en el factor donde teóricamente se esperaba que lo hicieran.

Identificamos dos tipos de riesgos: de alta (perder empleo o accidente de tráfico) y de baja probabilidad (incendio, infección alimentaria, atraco, sufrir una enfermedad grave o un error clínico). A su vez, diferenciamos entre pacientes que se consideran con mayor capacidad para afrontar riesgos (por ejemplo, ante pérdida de empleo, accidente de tráfico, incendio por cortocircuito e infección alimentaria), o con baja capacidad para afrontarlos (ante atraco, enfermedad grave o error clínico). Hay que señalar que cuando se controla la variable sexo hay riesgos que se valoran de forma diferente (p.e. la probabilidad de ser víctima de un atraco o un robo, es menos frecuente en hombres) aunque estas diferencias no afectan a la probabilidad de sufrir un error médico.

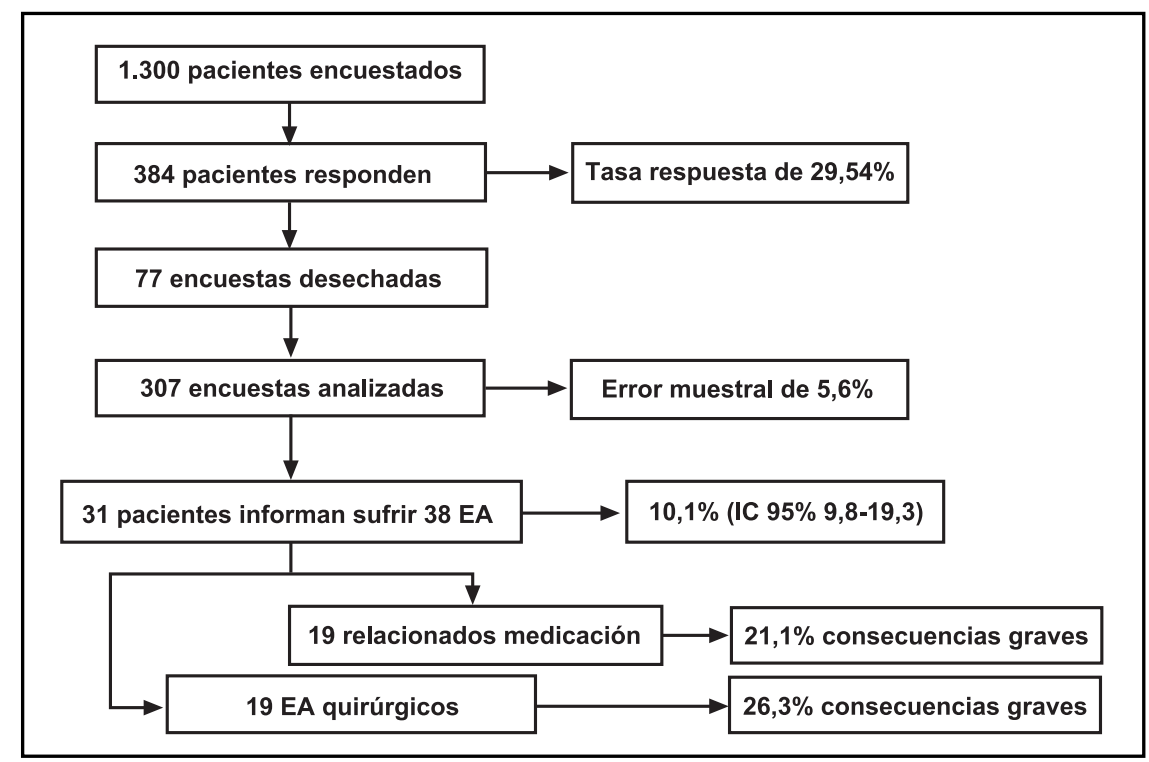

Figura 1. Frecuencia con la que se informa sufrir un EA. 


\section{Tabla 1. Estructura factorial del cuestionario de Percepción de Seguridad}

\begin{tabular}{|c|c|c|c|c|c|c|c|c|c|}
\hline & $\begin{array}{l}\text { Valor } \\
\text { propio }\end{array}$ & $\begin{array}{c}\text { Factor } \\
1^{\mathrm{a}}\end{array}$ & $\begin{array}{l}\text { Factor } \\
2^{\mathrm{b}}\end{array}$ & $\begin{array}{l}\text { Factor } \\
3^{c}\end{array}$ & $\begin{array}{l}\text { Factor } \\
4^{d}\end{array}$ & $\begin{array}{c}\text { Factor } \\
5^{\mathrm{e}}\end{array}$ & $\begin{array}{l}\text { Factor } \\
6^{f}\end{array}$ & $\begin{array}{l}\text { Factor } \\
7^{9}\end{array}$ & $\begin{array}{l}\text { Factor } \\
8^{\text {h }}\end{array}$ \\
\hline Confianza en enfermeros que le han cuidado & 0,8 & 0,9 & & & & & & & \\
\hline Confianza en auxiliares de enfermería que le han asistido & 0,7 & 0,8 & & & & & & & \\
\hline Confianza en médicos que le han atendido & 0,6 & 0,7 & & & & & & & \\
\hline Confianza en hospital donde ha ingresado & 0,5 & 0,6 & & & & & & & \\
\hline $\begin{array}{l}\text { Médicos y enfermeros han escuchado con atención } \\
\text { sus explicaciones }\end{array}$ & 0,7 & 0,6 & & & 0,5 & & & & \\
\hline Probabilidad de ser víctima de un incendio por cortocircuito & 0,6 & & 0,8 & & & & & & \\
\hline Probabilidad de ser víctima de una infección alimentaria & 0,6 & & 0,7 & & & & & & \\
\hline $\begin{array}{l}\text { Probabilidad de ser víctima } \\
\text { de un error médico con consecuencias graves }\end{array}$ & 0,7 & & 0,7 & & & & & & \\
\hline Probabilidad de ser víctima de un atraco/un robo & 0,6 & & 0,7 & & & & & & \\
\hline Probabilidad de ser víctima de una enfermedad grave & 0,6 & & 0,7 & & & & & & \\
\hline Capacidad para evitar una infección alimentaria & 0,6 & & & 0,8 & & & & & \\
\hline Capacidad para evitar que pierda el empleo & 0,7 & & & 0,7 & & & & & \\
\hline Capacidad para evitar un incendio por cortocircuito & 0,6 & & & 0,7 & & & & & \\
\hline Capacidad para evitar un accidente de tráfico & 0,7 & & & 0,5 & & & & 0,7 & \\
\hline $\begin{array}{l}\text { Explicado los posibles efectos y las precauciones } \\
\text { que debe tomar con el tratamiento }\end{array}$ & 0,8 & & & & 0,8 & & & & \\
\hline $\begin{array}{l}\text { Preguntado al médico sus dudas o preocupaciones } \\
\text { con respecto al tratamiento }\end{array}$ & 0,7 & & & & 0,8 & & & & \\
\hline $\begin{array}{l}\text { Errores clínicos son debidos a } \\
\text { fatiga o cansancio de los profesionales }\end{array}$ & 0,7 & & & & & 0,8 & & & \\
\hline $\begin{array}{l}\text { Errores clínicos son debidos a no disponer } \\
\text { de tiempo suficiente para todos los pacientes }\end{array}$ & 0,7 & & & & & 0,8 & & & \\
\hline $\begin{array}{l}\text { Errores clínicos son debidos a } \\
\text { insuficientes medios o recursos terapéuticos }\end{array}$ & 0,4 & & & & & 0,4 & & & \\
\hline Capacidad para evitar un atraco/un robo & 0,7 & & & & & & 0,8 & & \\
\hline $\begin{array}{l}\text { Capacidad para evitar } \\
\text { un error médico con consecuencias graves }\end{array}$ & 0,6 & & & & & & 0,7 & & \\
\hline Capacidad para evitar una enfermedad grave & 0,7 & & & & & & 0,6 & & $-0,4$ \\
\hline Probabilidad de perder su empleo & 0,6 & & & & & & & 0,7 & \\
\hline $\begin{array}{l}\text { Probabilidad de ser víctima } \\
\text { ser víctima de un accidente de tráfico }\end{array}$ & 0,5 & & & & & & & 0,5 & \\
\hline $\begin{array}{l}\text { Errores clínicos son debidos a } \\
\text { escasa formación de los profesionales sanitarios }\end{array}$ & 0,5 & & & & & & & & 0,6 \\
\hline Errores clínicos son debidos a desorganización en el Hospital & 0,5 & & & & & & & & 0,5 \\
\hline
\end{tabular}

aFactor 1: Confianza en médicos y enfermeros - varianza explicada 11,8\%. bFactor 2: Riesgos de baja probabilidad - varianza explicada 10,9\%. ${ }^{C}$ Factor 3: Alta capacidad para afrontar riesgo - varianza explicada 8,2\%. Factor 4: Comunicación positiva con el

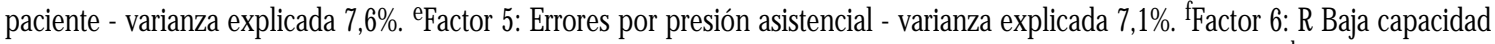
para afrontar riesgo - varianza explicada 7\%. 9Factor 7: Riesgos de alta probabilidad - varianza explicada 5,8\%. hFactor 8: Errores atribuibles a profesionales - varianza explicada 5,7\%. 
El análisis factorial de segundo orden (Tabla 2) dibuja una estructura de 2 dimensiones que explican hasta $48,71 \%$ de la varianza: confianza en profesionales y sistema sanitario, comunicación positiva con médico, y causas de errores clínicos; y probabilidad de determinados riesgos y capacidad percibida de evitarlos. El Alpha de Cronbach para los 26 elementos es de 0,7.

Cuando el paciente informa haber sufrido un EA (Tabla 3) se reduce significativamente su

Tabla 2. Estructura factorial del cuestionario de Percepción de Seguridad (factores de segundo orden)

\begin{tabular}{|lcc|}
\hline & Factor $\mathbf{1}^{\mathbf{a}}$ & ${\text { Factor } \mathbf{2}^{\mathbf{b}}}$ \\
\hline Errores atribuibles a profesionales & 0,7 & \\
Confianza en médicos y enfermeros & $-0,7$ & \\
Comunicación positiva con el paciente & $-0,7$ & \\
Errores por presión asistencial & 0,6 & 0,8 \\
Alta capacidad para afrontar riesgo & & 0,7 \\
Baja capacidad para afrontar riesgo & & 0,6 \\
Riesgos de alta probabilidad & & 0,5 \\
Riesgos de baja probabilidad & & \\
\hline
\end{tabular}

aFactor 1: Confianza y comunicación positiva con profesionales - 27,4\% de varianza explicada. bFactor 2: Percepción de seguridad clínica - 21,3\% de varianza explicada.

Tabla 3. Puntuaciones en los factores del cuestionario en función de haber sido víctima de un error y nivel de satisfacción

\begin{tabular}{|c|c|c|c|c|c|c|c|c|c|}
\hline & & $\begin{array}{l}\text { No informa } \\
\text { supuesto EA }\end{array}$ & $\begin{array}{c}\text { Informa } \\
\text { supuesto EA }\end{array}$ & $\mathrm{Chi}^{2}$ & $p=$ & $\begin{array}{c}\text { Baja } \\
\text { satisfacción }\end{array}$ & $\begin{array}{c}\text { Alta } \\
\text { satisfacción }\end{array}$ & $\mathrm{Chi}^{2}$ & $p=$ \\
\hline \multirow{3}{*}{$\begin{array}{l}\text { Confianza en médicos } \\
\text { y enfermeros }\end{array}$} & Baja & 1,4 & 25,9 & \multirow{4}{*}{50,9} & \multirow{3}{*}{0,0001} & 27,3 & 0,4 & \multirow{3}{*}{96,9} & \multirow{3}{*}{0,0001} \\
\hline & Media & 34,9 & 51,9 & & & 69,7 & 31,7 & & \\
\hline & alta & 63,7 & 22,2 & & & 3,0 & 67,9 & & \\
\hline \multirow{2}{*}{$\begin{array}{l}\text { Riesgos de baja } \\
\text { probabilidad }\end{array}$} & Bajo & 55,8 & 25,9 & & \multirow{3}{*}{0,003} & 37,5 & 55,1 & \multirow{3}{*}{3,5} & \multirow{3}{*}{0,063} \\
\hline & Medio & 44,2 & 74,1 & \multirow{2}{*}{8,6} & & 62,5 & 44,9 & & \\
\hline \multirow{2}{*}{$\begin{array}{l}\text { Alta capacidad de } \\
\text { afrontar riesgo }\end{array}$} & No & 67,5 & 56,5 & & & 74,1 & 66,0 & & \\
\hline & En parte & 32,5 & 43,5 & \multirow{2}{*}{1,1} & \multirow{2}{*}{0,292} & 25,9 & 34,0 & \multirow{2}{*}{0,7} & \multirow{2}{*}{0,402} \\
\hline \multirow{3}{*}{$\begin{array}{l}\text { Comunicación eficaz } \\
\text { con el paciente }\end{array}$} & No & 3,8 & 13,3 & & & 20,0 & 2,9 & & \\
\hline & Poco & 35,0 & 60,0 & \multirow{2}{*}{15,3} & \multirow{2}{*}{0,0001} & 71,4 & 32,2 & \multirow{2}{*}{48,7} & \multirow{2}{*}{0,0001} \\
\hline & SI & 61,2 & 26,7 & & & 8,6 & 64,9 & & \\
\hline \multirow{3}{*}{$\begin{array}{l}\text { Errores por presión } \\
\text { asistencial }\end{array}$} & Nunca & 35,5 & 26,9 & \multirow{3}{*}{0,7} & \multirow{3}{*}{0,69} & 16,1 & 37,6 & \multirow{3}{*}{6,4} & \multirow{3}{*}{0,042} \\
\hline & A veces & 57,7 & 65,4 & & & 71,0 & 56,3 & & \\
\hline & Mayoría veces & 6,8 & 7,7 & & & 12,9 & 6,1 & & \\
\hline \multirow{5}{*}{$\begin{array}{l}\text { Baja capacidad de } \\
\text { afrontar riesgo } \\
\text { Riesgos de alta } \\
\text { probabilidad }\end{array}$} & No & 92,5 & 92,3 & \multirow{3}{*}{0,1} & \multirow{3}{*}{0,965} & 90,9 & 92,6 & \multirow{3}{*}{0,1} & \multirow{3}{*}{0,729} \\
\hline & En parte & 7,5 & 7,7 & & & 9,1 & 7,4 & & \\
\hline & Bajo & 56,5 & 60,0 & & & 55,2 & 57,4 & & \\
\hline & Medio & 38,4 & 32,0 & \multirow{2}{*}{0,6} & \multirow{2}{*}{0,72} & 41,4 & 37,3 & \multirow{2}{*}{0,3} & 0861 \\
\hline & Alto & 5,1 & 8,0 & & & 3,4 & 5,3 & & \\
\hline Errores atribuibles & Nunca & 51,4 & 26,9 & & & 18,8 & 53,3 & & \\
\hline a los profesionales & A veces & 46,8 & 61,5 & 116 & 00 & 71,9 & 44,8 & 164 & 0001 \\
\hline & Mayońa veces & 1,8 & 11,5 & 11,0 & 0,003 & 9,4 & 1,9 & 10,4 & 0,0001 \\
\hline
\end{tabular}


confianza en profesionales y sistema sanitario ( $p<0,001$ ), es más probable que considere inapropiada la comunicación con el médico ( $p=0,0001$ ), atribuya más fácilmente los errores a los profesionales ( $p=0,003$ ) y vea incrementada la probabilidad de los riesgos considerados de menor frecuencia por otros encuestados (donde se incluye la probabilidad de sufrir un error clínico). La capacidad para evitar un error no se ve afectada $(p=0,292$ o $p=0,965)$. La satisfacción del paciente actúa como variable moduladora de estos resultados (Tabla 3). En este caso, observamos que la confianza disminuye de forma significativa únicamente en el caso de los pacientes insatisfechos ( $p=0,049$ ). El mismo efecto se observa a la hora de considerar la probabilidad de los riesgos considerados a priori de baja probabilidad: los insatisfechos consideran estos riesgos más probables ( $p=0,005)$.

La Tabla 4 muestra los resultados desagregados. La probabilidad de sufiri un emor clínico se incremen-

Tabla 4. Capacidad discriminante de los elementos de la escala en función de si el paciente informa haber sufrido un error clínico

\begin{tabular}{|c|c|c|c|}
\hline & $\begin{array}{l}\text { No informa de } \\
\text { supuesto EA }\end{array}$ & $\begin{array}{l}\text { Informa de } \\
\text { supuesto EA }\end{array}$ & $p=$ \\
\hline Confianza en enfermeros que le han cuidado & 82,5 & 69,0 & 0,0001 \\
\hline Confianza en auxiliares de enfermería que le han asistido & 78,4 & 58,6 & 0,012 \\
\hline Confianza en médicos que le han atendido & 88,1 & 53,6 & 0,001 \\
\hline Confianza en hospital donde ha ingresado & 73,5 & 31,0 & 0,001 \\
\hline Médicos y enfermeros han escuchado con atención sus explicaciones & 82,7 & 53,3 & 0,001 \\
\hline Probabilidad de ser víctima de un incendio por cortocircuito & 5,4 & 0,0 & 0,040 \\
\hline Probabilidad de ser víctima de una infección alimentaria & 8,8 & 6,9 & 0,501 \\
\hline Probabilidad de ser víctima de un error médico con consecuencias graves & 5,8 & 10,3 & 0,002 \\
\hline Probabilidad de ser víctima de un atraco/un robo & 21,5 & 27,6 & 0,532 \\
\hline Probabilidad de ser víctima de una enfermedad grave & 20,3 & 31,0 & 0,229 \\
\hline Capacidad para evitar una infección alimentaria & 10,6 & 7,4 & 0,363 \\
\hline Capacidad para evitar que pierda el empleo & 14,8 & 12,0 & 0,136 \\
\hline Capacidad para evitar un incendio por cortocircuito & 11,1 & 3,8 & 0,034 \\
\hline $\begin{array}{l}\text { Explicado los posibles efectos y las precauciones } \\
\text { que debe tomar con el tratamiento }\end{array}$ & 79,7 & 45,2 & 0,0001 \\
\hline $\begin{array}{l}\text { Preguntado al médico sus dudas o preocupaciones } \\
\text { con respecto al tratamiento }\end{array}$ & 68,9 & 45,2 & 0,021 \\
\hline Errores clínicos son debidos a fatiga o cansancio de los profesionales & 17,0 & 35,7 & 0,044 \\
\hline $\begin{array}{l}\text { Errores clinicos son debidos a } \\
\text { no disponer de tiempo suficiente para todos los pacientes }\end{array}$ & 46,7 & 60,0 & 0,385 \\
\hline Errores clínicos son debidos a insuficientes medios o recursos terapéuticos & 20,0 & 7,7 & 0,160 \\
\hline Capacidad para evitar un atraco/un robo & 2,5 & 3,6 & 0,942 \\
\hline Capacidad para evitar un error médico con consecuencias graves & 3,0 & 3,6 & 0,623 \\
\hline Capacidad para evitar una enfermedad grave & 6,3 & 11,1 & 0,498 \\
\hline Capacidad para evitar un accidente de tráfico & 9,8 & 0,0 & 0,106 \\
\hline Probabilidad de perder su empleo & 10,1 & 16,0 & 0,651 \\
\hline Probabilidad de ser víctima de un accidente de tráfico & 26,3 & 24,1 & 0,844 \\
\hline Errores clínicos son debidos a escasa formación de los profesionales sanitarios & 4,7 & 15,4 & 0,075 \\
\hline Errores clínicos son debidos a desorganización en el Hospital & 21,6 & 58,6 & 0,0001 \\
\hline
\end{tabular}


ta cuando se informa de un EA ( $p=0,002)$, pero no se incrementa la capacidad para evitarlo $(p=0,623)$.

\section{Discusión}

Este estudio corresponde a una línea de investigación sobre percepción de los pacientes de los errores clínicos ${ }^{17}$ que, en definitiva, busca implicarlos en las distintas fases del proceso asistencial, incluida la toma de decisiones clínicas, como estrategia para incrementar la seguridad.

Existen distintos enfoques a la hora de preguntar a los pacientes si han sufrido un $\mathrm{EA}^{8,10,12}$. En este caso, se optó por utilizar el término error (aún siendo conscientes de que no todos los errores son EA ni todos los EA errores), por acortar el tiempo y por diferenciar entre errores de medicación o de la cirugía.

Los datos confirman que se considera improbable ser víctima de un error y que esa percepción de riesgo se incrementa tras sufrir un EA. Falta de tiempo, fatiga de los profesionales y fallos organizativos son las causas fundamentales de los errores para los encuestados, coincidiendo con el estudio Alberta ${ }^{12}$. Muchos pacientes desconocen que su actitud frente a los tratamientos puede reducir el riesgo de un error clínico. Este dato resulta relevante cuando desde distintas Administraciones Sanitarias se están implantando campañas informativas/formativas dirigidas a los ciudadanos (p.e. Ministerio de Sanidad y Consumo, SESCAM, SERMAS, etc.).

Los datos de este estudio sugieren que la confianza y la comunicación positiva con el médico están estrechamente relacionadas y contribuyen a evitar, en caso de EA, la pérdida de

\section{REFERENCIAS}

1. Stelfox HT, Palmisani S, Scurlock C, Orav Ej, Bates DW. The «o Err is Human» report and the patient safety literature. Qual Saf Health Care 2006; 15: 174-8.

2. Aranaz-JM, Aibar-C, Vitalier-J, Ruiz-López P, Limón-Ramírez R, TEROL-García E, AND tHE ENEAS Work Group. Incidence of adverse events related to health care in Spain: results of the Spanish National Study of Adverse Events. J Epidemiol Community Health 2008; 62: 1022-9. confianza en los profesionales y en el hospital, en línea con lo sugerido por otros estudios $12,19,20$.

La frecuencia de EA informado por los pacientes está en consonancia con otros resultados. Ahora bien, el número de informantes que suman más de un EA es inferior al esperable según el estudio ENEAS (18\%). Es difícil interpretar este resultado, pero pudiera suponer que este aspecto no se recoge adecuadamente en el cuestionario. Esta no es la única limitación del estudio. A diferencia del estudio Alberta no incluimos ninguna pregunta sobre si creía el paciente que en caso de un error los profesionales le informarían. Por otro lado, aunque en España es normal una tasa de respuesta inferior a $30 \%$, hubiera sido deseable superar esa tasa. Por último, no se consideraron características de personalidad de los informantes o si cursan con una enfermedad aguda o crónica. Estos aspectos pudieran ser relevantes y merecería la pena que fueran analizados en futuros estudios.

Cuando se tiene sensación de riesgo es más probable que se actúe para minimizarlo ${ }^{21}$, pero cuando esa sensación es exagerada puede tener el efecto contrario, máxime en el caso de los errores clínicos. Este estudio, confirma esta impresión y pone de relieve la importancia de actuar con el paciente que ha sufrido un EA para minimizar futuras consecuencias negativas en su relación con cualquier unidad del Sistema Sanitario.

Reducir los EA es un objetivo de la práctica total de los Servicios de Salud. Hasta la fecha, la mayoría de las acciones han estado orientadas hacia los profesionales pero, cada vez es más frecuente, encontrar acciones dirigidas a los pacientes. Este cuestionario puede contribuir al diseño de las actuaciones en pro de la seguridad que buscan implicar a los pacientes. 
Problems and Harms in Primary Health Care. Ann Fam Med 2004; 2: 333-40.

6. Mira JJ, Aranaz JM, Vitaller J, Ziadi M, Lorenzo S, Rebasa P et al. Percepción de seguridad clínica tras el alta hospitalaria. Med Clin (Barc) 2008; 131(Supl 3): 26-32.

7. Adams RE, Boscarino JA. A community survey of medical errors in New York. Int J Qual Health Care 2004; 16: 353-62.

8. Agoritsas T, Bovier PA, Perneger TV. Patient Reports of Undesirable Events During Hospitalization. J Gen Intern Med 2005; 20: 922-8.

9. Vincent CA, Pincus T, ScurR JH. Patients' experience of surgical accidents. Qual Saf Health Care 1993; 2: 77-82.

10. Blendon RJ, DesRoches CM, Brodie M, Benson JM, ROSEN AB, SCHNEIDER E ET AL. Views of Practicing Physicians and the Public on Medical Errors. N Engl J Med 2002; 347: 1933-40.

11. COMMISSION EUROPÉENNE. Les erreurs médicales. Eurobaromètre Special 241. Janvier 2006. Disponible en: http:// europa.eu.int/comm/health/ph_information/ documents/eb_64_fr.pdf [consultado 2 de febrero de 2009].

12. Northcott H, Vanderheyden L, Northcott J, Adair C, McBrien-Morrison Ch, Norton P, Cowell J. Perceptions of preventable medical errors in Alberta, Canada. Int J Qual Health Care 2008; 20: 115-22.

13. Evans SM, Berry JG, Smith BJ, Ester AJ. Consumer perceptions of safety in hospitals. BMC Public Health, 2006; 6: 41. Disponible en: http:// www.biomedcentral.com/1471-2458/6/41/prepub. [consultado 2 de febrero de 2009].

14. Kaiser Family Foundation. National survey on Americans as health care consumers: an update on the role of quality information. Menlo Park, CA: Kaiser
Family Foundation/Agency for Healthcare Research and Quality, 2000. Available from http:// www.kff.org/kaiserpolls/3093-index.cfm [consultado 12 de enero de 2009].

15. Ministerio Sanidad y Consumo. Barómetro Sanitario. 2008. Disponible en: http://www.msc.es/estadEstudios/estadisticas/inforRecopilaciones/barometro/ home.htm. [consultado 19 febrero de 2009].

16. Massó P, Aranaz J, Mira JJ, Perdiguero E, Aibar C. Adverse events in hospitals: The patient's point of view. Quality \& Safety Health Care 2009; (en prensa).

17. Mira JJ, Aranaz J. Vitaller J, Lorenzo S. Patients' Perception of Hospital Safety. En: Øvretveit J, Sousa P (Eds). Quality and Safety Improvement Research: methods and research practice from the international quality improvement research network (QIRN). Escola Nacional de Saúde Pública and MMC Karolinska Institutet. Lisboa 2008.

18. Pérez-Jover V, Tomás O, Mira JJ, Rodríguez-Marín J. Sistemas de evaluación, valoración de la calidad percibida y satisfacción. Cuestionarios de medida. En: Aranaz JM, Aibar C, Vitaller J, Mira JJ (Directores). Gestión Sanitaria. Calidad y seguridad de los pacientes. Díaz de Santos. Madrid 2008.

19. Vanderheyden LC, Northcott HC, Adair CE, BrienMorrison C, Meadows LM, Norton P et al. Reports of preventable medical errors from the Alberta Patient Safety Survey 2004. Healthc Q 2005; 8 Spec No: 107-14.

20. Mira JJ, Aranaz J, Vitalier J, Gea MT. Percepción de seguridad de los pacientes de cirugía ambulatoria. Cir Esp 2009. doi:10.1016/j.ciresp.2008.07.009.

21. Peters E, Slovic P, Hibbard JH, Tusler M. Why worry? Worry, risk perceptions, and willingness to act to reduce medical errors. Health Psychol 2006; 25: 144-52. 